\title{
The Impact of Different Lung Ultrasound Protocols in the Assessment of Lung Lesions in COVID-19 Patients: Is There an Ideal Lung Ultrasound Protocol?
}

\author{
Yale Tung-Chen ${ }^{1,6}$ (D) Silvia Ossaba-Vélez ${ }^{2} \cdot K^{2}$ vin Stephen Acosta Velásquez ${ }^{2} \cdot$ Maria Luz Parra-Gordo $^{2}$. \\ Aurea Díez-Tascón ${ }^{2}$ · Tomás Villén-Villegas ${ }^{3}$ - Esther Montero-Hernández ${ }^{1}$ - Andrea Gutiérrez-Villanueva ${ }^{1}$. \\ Ángela Trueba-Vicente ${ }^{4} \cdot$ Isabel Arenas-Berenguer ${ }^{5} \cdot$ Milagros Martí de Gracia $^{2}$
}

Received: 9 May 2021 / Accepted: 21 June 2021 / Published online: 2 December 2021

(c) Società Italiana di Ultrasonologia in Medicina e Biologia (SIUMB) 2021

\begin{abstract}
Background In the past months, several lung ultrasonography (LUS) protocols have been proposed, mainly on previously validated schemes independent of coronavirus disease 2019 (COVID-19).

Objectives The main purpose of this study was to determine the impact and accuracy of different LUS protocols proposed in COVID-19.

Methods Patients were evaluated with a standard sequence of LUS scans in 72 intercostal spaces along 14 anatomic lines in the chest. A scoring system of LUS findings was reported and then analyzed separately according to each proposed LUS protocol zones. This score was then correlated to a validated Pulmonary Inflammation Index (PII) on chest Computed Tomography (CT).

Results Thirty-two patients were enrolled. The most frequent pattern was ground-glass opacities in the chest X-ray (53.1\%), chest CT $(59.1 \%)$ and subpleural or lobar consolidations $(40.8 \%)$ in the posteroinferior areas $(p<0.001)$ on LUS. The Interclass Correlation Coefficient (ICC) was significantly correlated with almost every protocol analyzed except the 8-zone $(p=0.119)$ and the 10-zone protocol that only included one posterior point $(p=0.052)$. The highest ICC was obtained with a 12-zone protocol (ICC $0.500 ; p=0.027$ ) and decreased as more points were included.

Conclusions In conclusion, our study results suggest that performing an ultrasound protocol with 12-zone scanning, including the superior and inferior areas of the anterior, lateral and posterior regions of the chest was consistent with higher ICC and higher degree of concordance with CT. We emphasize the need of a more standardization technique to further implement and develop this imaging modality in COVID-19.
\end{abstract}

Keywords Point-of-care ultrasonography (POCUS) · Lung ultrasonography (LUS) · Coronavirus disease 2019 (COVID19) $\cdot$ Severe acute respiratory syndrome coronavirus 2 (SARS-CoV-2)

\section{Introduction}

Ten months after the declaration of a global pandemic due to the novel coronavirus disease (COVID-19), more than 100 million confirmed cases and over 2.3 million deaths have been reported globally [1].

These facts enhance the critical need for an accessible, reproducible, low-cost and safe imaging modality for the

Yale Tung-Chen

yale.tung@salud.madrid.org

Extended author information available on the last page of the article diagnosis, risk stratification, monitoring and management of COVID-19 patients. Lung ultrasonography (LUS) qualifies for such purpose, providing an evidence-based method which will aid to diagnose and stratify patients, as well as guide therapy and early detect complications [2].

However, contrasting the relevant role of LUS, there is no validated scanning protocol in COVID-19 patients. At the moment, the main recommendation is to use previously validated schemes in other conditions other than COVID-19.

In the past months, several imaging protocols had been proposed, based on the number of areas or points to explore (see Table 1): 8-zone protocol [3], 10-zone protocol [4, 5], 12-zone protocol [6, 7], 14-zone protocol [8], 16-zone 





protocol [9] and 18-zone protocol [10] and 72-zone protocol [11].

Finding the optimal balance between the acquisition time and accuracy represents one of the main challenges. Whenever possible, a lower number of acquisition areas is preferred, reducing the cross-contamination and exposition of healthcare professionals, but this could lead to underestimations.

Additionally, the development of a more standardized approach will allow comparisons and reproducibility across different studies and exams, as well as facilitate research on pattern recognition with artificial intelligence algorithms and telematic applications [8].

To our best knowledge, the formulation of such an approach is in need for more research. We aimed to determine the impact on accuracy of currently proposed LUS scanning protocols, in determining the presence and extension of lung lesions, in patients with confirmed COVID-19.

\section{Patients and Methods}

This was a prospective study performed in the emergency department (ED) of an academic hospital, conducted in accordance with the Declaration of Helsinki, and approved by the Research Ethics Committee of our University Hospital. We obtained informed consent from each patient.

\section{Patient Selection}

Patients admitted to the ED with a confirmed COVID-19 requiring a chest computed tomography (CT) for evaluation were included. We defined a confirmed case as any patient with clinical symptoms and positive reverse transcription polymerase chain reaction (RT-PCR) or antigen test for the severe acute respiratory syndrome coronavirus 2 (SARS-CoV-2).

The main indication for CT was a negative chest X-ray, respiratory failure (oxygen saturation below $92 \%$ ) or high suspicion of pulmonary embolism (PE).

We excluded patients $<18$ years or those who declined to participate. A sample of patients who met these inclusion criteria were enrolled and prospectively studied.

\section{Study Protocol}

Patients underwent a chest CT performed by fellowshiptrained radiologists together with a LUS, performed by an ultrasound fellowship-trained internal medicine physician performed all the ultrasound exams. Therefore, an opportunity sampling method was implemented for patient selection.

\section{Chest CT Data Collection}

Non-contrast chest CT scans were obtained by a multidetector CT (SOMATOM go.Up, Siemens Healthliners, Erlangen, Germany). The examination was performed with the patient in the supine position and at the end of inspiration. Scans were acquired and reconstructed as axial images using the following parameters: $1.5-\mathrm{mm}$ section thickness, $0.7-\mathrm{mm}$ interval, $130 \mathrm{kVp}$. A low-dose protocol was implemented with a mean CT Dose Index volume (CTDIvol) of $2 \mathrm{mGy}$.

Blinded to the clinical information, one radiologist trainee with 2 years of experience (KSAV) and four senior radiologists with more than 10 years of experience (MMG, ADT, MLGP, SOV) reviewed all the images.

Images were interpreted using the lung and mediastinum window settings. Each lung was divided into 10 zones based on the anatomy of the lung segments (Fig. 1). The presence and distribution of the following radiological abnormalities were evaluated in each lung segment [12]: ground-glass opacities (GGO), interlobular septal thickening, crazy paving pattern, consolidation, subpleural line, pleural thickening and effusion.

\section{Ultrasound Data Collection}

The ultrasound exam was performed with the patient in supine or near-supine position for the anterior scanning, and in the sitting or lateral decubitus position for the posterior scanning.

The probe was positioned obliquely, along the intercostal spaces [13]. The LUS examination was obtained moving the probe along anatomical reference lines (Fig. 2), 2nd-4th intercostal space (ICS) of parasternal, midclavicular, anterior axillary and midaxillary line (on the right side to the 5th ICS), whereas for the posterior chest, the paravertebral (2nd-10th ICS), sub-scapular (7th-10th ICS) and posterior axillary (2nd-10th ICS) lines [11]. A video clip was recorded along each anatomical line, recording at least $3 \mathrm{~s}$ at each ICS.

The examinations were performed using a GE VENUE ultrasound system fitted with a phased and curvilinear array transducer (1.5-4.5 MHz) (General Electrics Healthcare, Madrid, Spain). The sonographer was blinded to the patient's past medical history, vital signs, symptoms, laboratory measurements, CT scan results and therapy.

In our limited experience with COVID-19 patients, it takes less than 10 min to perform LUS, excluding cleaning time [7].

\section{Outcome Measures and Definitions}

The main purpose of this study was to determine the impact and accuracy of different LUS protocols and the correlation 

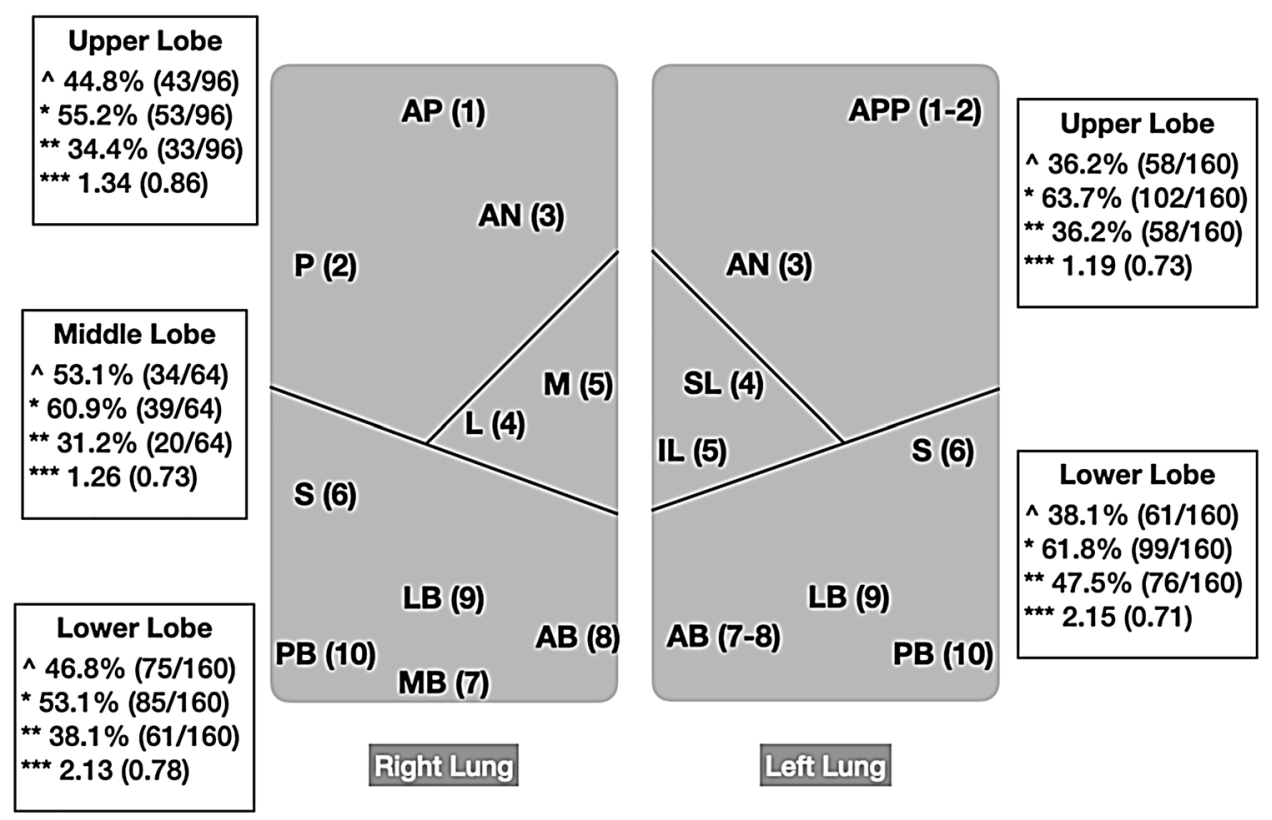

Fig. 1 Bronchopulmonary segments (10 per hemithorax). Right lung. Upper lobe: AP (1)—apical; P (2)—posterior; AN (3)—anterior. Middle lobe: L (4)-lateral; M (5)-medial. Lower lobe: S (6)superior; MB (7)—medial; AB (8)—anterior; LB (9)-lateral; PB (10) — posterior. Left lung. Upper lobe: APP (1-2)—apicoposterior; AN (3)—anterior; SL (4)—superior lingula; IL (5)—inferior lingula.

Lower lobe: S (6)—superior; AB (7-8) Anteromedial; LB (9)—lateral; PB (10)—posterior. Findings in each lobe: normal (percentage of normal lobes in our cohort); *ground-glass opacities (percentage of affected lobes in our cohort); **consolidation (percentage lobes affected in our cohort); ***average lung score (standard deviation)

Fig. 2 Anatomical reference lines of the Lung Ultrasonography (LUS) protocol. Second to fourth intercostal space of parasternal (dashed line), midclavicular (solid line), anterior axillary (solid line) and midaxillary (dashed) line (on the right side to the fifth). Second to tenth intercostal space of the paravertebral (solid line) and posterior axillary (dash-dotted) lines. Seventh to tenth intercostal space of the sub-scapular (dashed) line
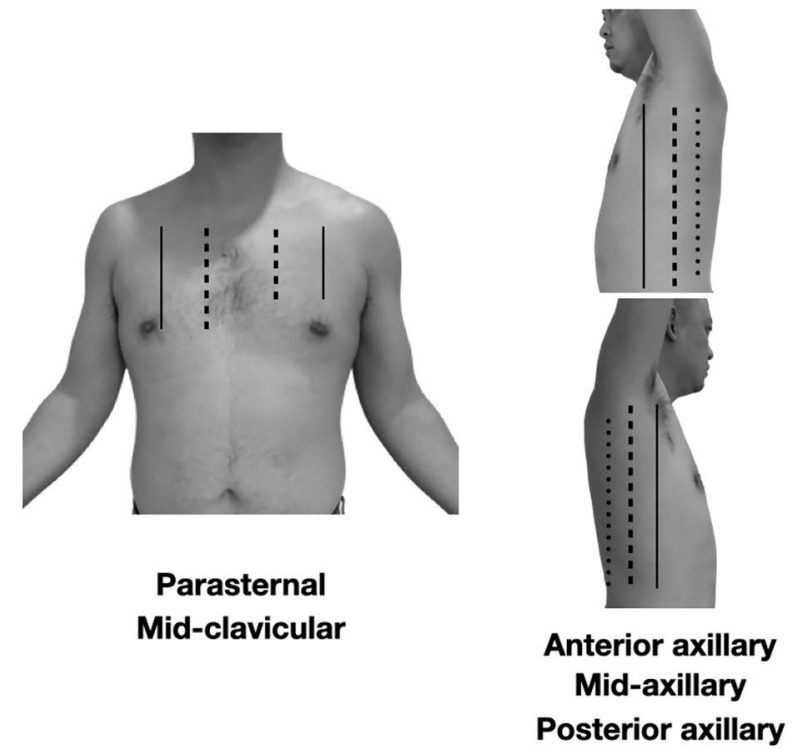

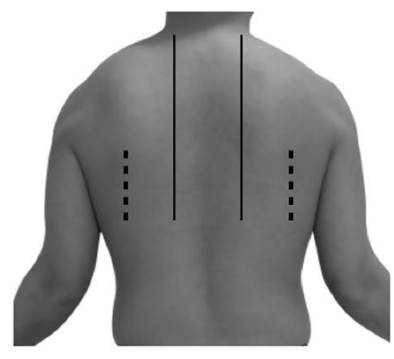

Subscapular Paravertebral with the PII in the chest $\mathrm{CT}$ in patients with confirmed coronavirus disease (COVID-19).

According to the evaluation criterion established by Chongqing Radiologist Association of China, the pulmonary inflammation index (PII) was obtained from each patient [14]. PII $=($ distribution score + size score $) / 40 \times 100 \%$. Distribution score: scored according to the lesion distribution, one score for each lung segment, and 20 scores for left and right lung. Size score: according to whether the lesion occupied $\geq 50 \%$ (score of 1 ) or $<50 \%$ (score of 0 ).

We developed a numeric Lung Score based on the pathological findings in each lung sonographic area. We differentiated the lung scanning areas (Fig. 2) of each protocol and gave each pathological finding (Table 2) a different score: 
Table 2 Definition and interpretation of the main findings on Lung Ultrasonography in COVID-19

\begin{tabular}{ll}
\hline LUS finding & Definition \\
\hline A-lines & Horizontal reverberation artifacts parallel to the pleural line \\
B-lines & $\begin{array}{c}\text { Hyperechoic vertical artifacts that arise from the pleural line, extending to the bottom of } \\
\text { the screen without fading that erases the A-line artifact } \\
\text { Multiple converging or coalescent B-lines }\end{array}$ \\
Confluent & Discrete, well demarcated B-lines \\
Isolated & Indented or broken pleural line \\
Irregular Pleural Line & Anechoic space between the parietal and visceral pleura \\
Pleural effusion & Hypoechoic areas smaller than $1 \mathrm{~cm}$ in diameter, surrounded by a hyperechoic artifact tail \\
Subpleural consolidation &
\end{tabular}

LUS lung ultrasound

- Irregular pleural line or focal B lines: 1 point

- Confluent B lines: 2 points

- Subpleural or lobar consolidation or pleural effusion: 3 points

We summed every area's points, dividing into the maximum score (i.e., for a 10-zone protocol, the maximum score would be 3 points $\times 10$ zones $=30$ ) and multiplying by 100 obtaining the patient's standardized Lung Score according to the assessed protocol, ranging from 0 to 100 .

\section{Statistical Analysis}

Baseline characteristics are presented as mean and standard deviation (SD) for continuous variables and count and proportions for categorical variables.

The interclass correlation coefficient (ICC) was used to assess the degree of agreement between the LUS score of each protocol and the PII on chest CT. A sample of 30 patients was obtained as previously recommended in reliability studies [15]. An ICC of less than 0.50 was considered poor, from 0.50 to 0.75 moderate, $0.75-0.90$ was considered good and $0.90-1$ excellent [15].

We assumed an $\alpha$-value of 0.05 for two-sided hypothesis testing. Analyses were conducted with the statistical IBM SPSS software v25.0 (SPSS Inc., Chicago, IL, USA).

\section{Results}

A total of 32 patient were enrolled between September and October 2020 (summarized in Table 3). The mean age was 67.9 years (SD 19.5) and 59.4\% were female. 18 patients $(56.2 \%)$ had an underlying comorbidity. They had an average of 8.03 days (SD 4.14) after symptom onset. The patients were normotensive and had low oxygen saturation $(92.3 \%$-SD 4.1 , with a respiratory rate of $25.6 \mathrm{rpm}-\mathrm{SD} 1.2$ ). The mean lymphocyte count was
$1.21 \times 10^{9}$ (SD 0.52, normal value-NV: $\left.1.0-4.0\right)$, CRP was $83.7 \mathrm{mg} / \mathrm{dL}$ (SD 87.2, NV: 0-10), D-dimer $2244 \mathrm{ng} /$ $\mathrm{mL}$ (SD 4156, NV: <400) and LDH was $312.8 \mathrm{U} / \mathrm{L}$ (SD 87.2; NV: 140-280) at admission.

Regarding the imaging studies, all the included patients had a chest X-ray, CT and underwent a LUS study (see Table 4 and Fig. 3). The most frequent pattern was groundglass opacities (GGOs) in the chest X-ray $(53.1 \%$ [17 patients out of 32]) and chest CT (59.1\% [378 lobes affected out of 640 explored]). About the LUS, the most common findings were subpleural or lobar consolidations (40.8\% [940 ICS out of 2304 explored]) in the posteroinferior areas $(p<0.001)$ (see Fig. 1).

The average PII value was $43.8 \%$ (SD 19.9). Interclass Correlation analysis (see Table 5) showed that the PII was significantly correlated with almost every protocol analyzed except the 8-zone $(p=0.119)$ and the 10-zone protocol with only one posterior point scanned $(p=0.052)$. The highest ICC was obtained with a 12-zone protocol (ICC 0.500; $p=0.027)$ and decreased as more scanning points were included.

\section{Discussion}

Easy to access and reliable diagnostic methods which can accurately guide management in COVID-19 are vital in nonhospital settings and areas with limited resources. Some studies start to point out that LUS could be a first-line diagnostic tool alternative to conventional chest $\mathrm{X}$ ray and $\mathrm{CT}$ scan, including the critically ill patients, where LUS score had been already been proven to be useful [16]. Moreover, since there is no exposure to ionizing radiation, can be considered in vulnerable population such as pregnant women and children $[17,18]$.

In this pandemic context, a useful proposal for LUS standardization in COVID-19 was reported, defining different parameters such as the acquisition time, the type of 
Table 3 Demographics and clinical characteristics of patients included $(\mathrm{N}=32)$

\begin{tabular}{|c|c|}
\hline Demographics & \\
\hline Gender (female) $-N(\%)$ & $19(59.4)$ \\
\hline Age (years) mean (SD) & $67.9(19.5)$ \\
\hline Caucasic $-N(\%)$ & $18(56.3)$ \\
\hline Past medical history-N(\%) & \\
\hline Pulmonary disease & $5(15.7)$ \\
\hline Diabetes Mellitus & $6(18.8)$ \\
\hline Hypertension & $13(40.6)$ \\
\hline Dyslipidemia & $13(40.6)$ \\
\hline Obesity & $3(9.4)$ \\
\hline Heart disease & $10(31.3)$ \\
\hline Previous thromboembolic disease & $3(9.4)$ \\
\hline Dementia & $4(12.5)$ \\
\hline Mean days from Symptom Onset & $8.03(4.14)$ \\
\hline Phyisical exam & \\
\hline $\mathrm{SBP}(\mathrm{mmHg})$ mean $(\mathrm{SD})$ & $127.3(17.2)$ \\
\hline Heart rate (rpm) mean (SD) & $79.8(18.2)$ \\
\hline Respiratory rate (rpm) mean (SD) & $25.6(1.2)$ \\
\hline Temperature $\left({ }^{\circ} \mathrm{C}\right)$ mean $(\mathrm{SD})$ & $36.2(1.0)$ \\
\hline $\mathrm{SO}_{2}(\%)$ mean $(\mathrm{SD})$ & $92.3(4.1)$ \\
\hline Weight (kg) mean (SD) & $73(12.38)$ \\
\hline Height (cm) mean (SD) & $158(8.04)$ \\
\hline Laboratory results-mean $(S D)$ & \\
\hline Hemoglobin—g/dL & $13.7(1.9)$ \\
\hline $\mathrm{WBC} \times 109 / \mathrm{L}$ & $8046.9(4545.3)$ \\
\hline Lymphocite $\times 10$ 9/L & $1209.1(520.5)$ \\
\hline $\mathrm{LDH}-\mathrm{U} / \mathrm{L}$ & $312.8(102.6)$ \\
\hline Platelets $\times 103 / \mathrm{L}$ & $247.2(90.7)$ \\
\hline D-Dimer-ng/mL & $2244.2(4156.3)$ \\
\hline Creatinine $-\mathrm{mg} / \mathrm{dL}$ & $0.92(0.35)$ \\
\hline ALT-IU/L & $46.3(30.8)$ \\
\hline C-reactive protein $-\mathrm{mg} / \mathrm{dL}$ & $83.7(87.2)$ \\
\hline Therapy $-N(\%)$ & \\
\hline Remdesivir & $2(6.3)$ \\
\hline Corticosteroids & $21(65.6)$ \\
\hline LMWH—prophylaxis & $17(53.2)$ \\
\hline LMWH-intermediate & $3(9.3)$ \\
\hline LMWH-therapeutic & $5(15.6)$ \\
\hline Antibiotic & $14(43.8)$ \\
\hline Follow-up $-N(\%)$ & \\
\hline ICU admission & $3(9.4)$ \\
\hline Increase requirement of oxygen & $8(25)$ \\
\hline Mortality & $3(9.4)$ \\
\hline
\end{tabular}

$A L T$ alanine transaminase, $I C U$ intensive care unit, $L D H$ lactate dehydrogenase, $L M W H$ low molecular weight heparin, rpm rate per minute, $S D$ standard deviation, $W B C$ white blood cell

transducer, the imaging settings and the scanning points [8], mainly based on expert's recommendation, with scarce adoption.
Table 4 Imaging modalities (chest computed tomography, X-ray and Point-of-care ultrasound) findings of patients included $(N=32)$

\section{Chest computed tomography}

Total zones (10 per hemithorax, 20 per patient, $N=640) \quad 640(100)$

Normal zones $(N=640)$

$177(27.7)$

Abnormal findings

GGO $(N=640)$

$378(59.1)$

Consolidation $(N=640)$

247 (38.6)

Pleural effusion $(N=32)$

5 (15.6)

Subpleural line $(N=640)$

$99(15.5)$

Crazy paving pattern $(N=640)$

$39(6.1)$

Interlobular septal thickening $(N=640)$

27 (4.2)

Atelectasis $(N=640)$

Pleural thickening $(N=640)$

PII \% (SD)

$43.8(19.9)$

Chest $X$-ray-N $(\%)$

32 (100)

4 (12.5)

Abnormal findings

Ground-glass opacity (GGO)

$17(53.1)$

Interstitial pattern

$11(34.4)$

Bilateral

$23(71.9)$

Unilateral

5 (15.6)

Point-of-care ultrasonography (POCUS) results

Total ICS explored (32 patients, 72 ICS per patient, $N=2304$ )

Normal zones $(N=2304)$

$2304(100)$

Abnormal findings

Consolidation $(N=2304)$

$535(23.2)$

Pleural effusion $(N=32)$

$940(40.8)$

Confluent B-lines zones $(N=2304)$

$10(31.3)$

Irregular pleural line or isolated B-lines zones $(N=2304)$

GGO ground-glass opacity, ICS intercostal space, PII: Pulmonary Inflammation Index, POCUS point-of-care ultrasonography, $S D$ standard deviation

Subsequently, several imaging protocols had been proposed, based on the number of areas or points to explore (see Table 1), complicating the reproducibility of the results and research.

In our study, we selected PII as the CT score index comparator. PII is a validated index that in previous studies significantly correlated with the degree of pulmonary inflammation and the main clinical symptoms and laboratory results [14]. This would more appropriately address the pathophysiology of the disease, beyond the anatomic abnormalities, at a cost of weaker correlations than reported previously in the literature [6].

Our findings are comparable to a recently published study on this matter [19]. In this multicentric study, they analyzed the outcome of 3 different LUS imaging protocols $(4,8$ and 12-zone acquisitions) from 88 patients, comparing it to the 

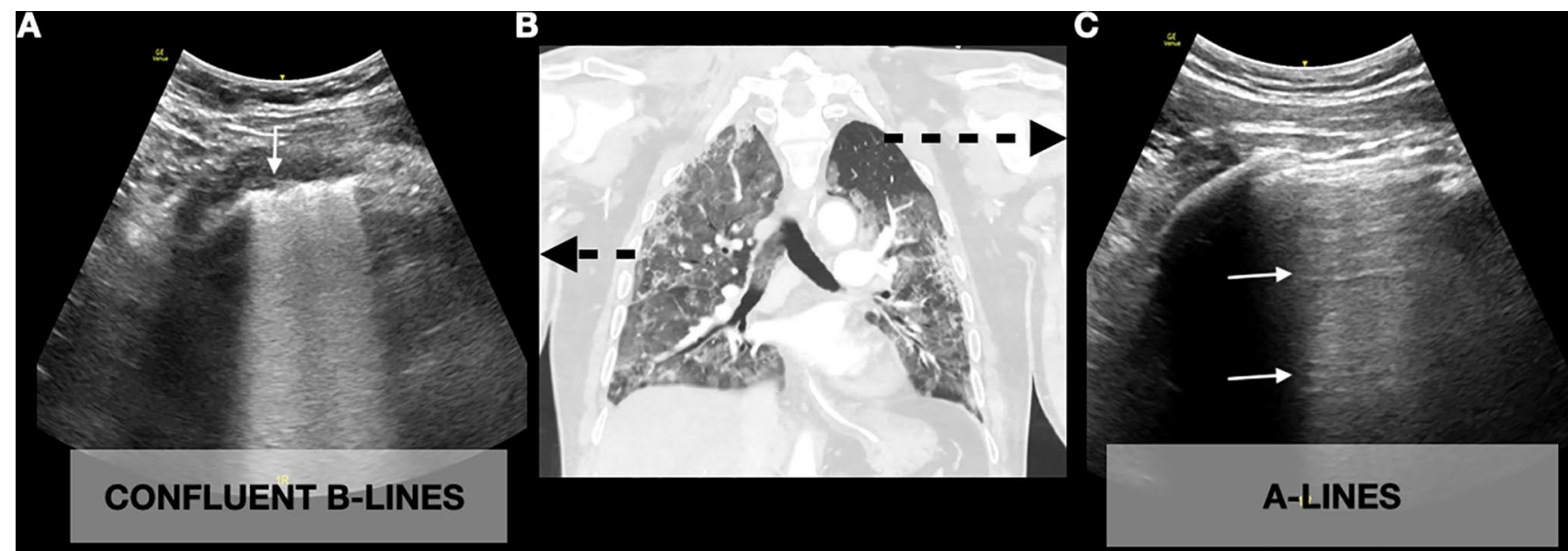

D

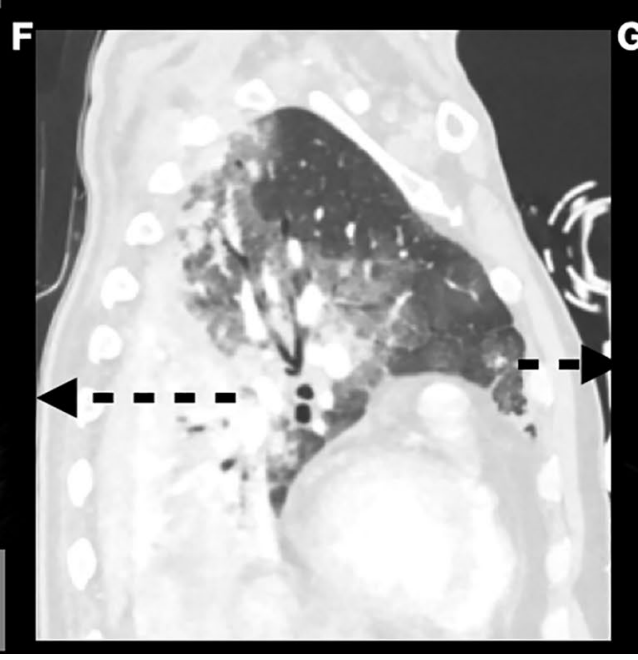

\section{A-LINES}

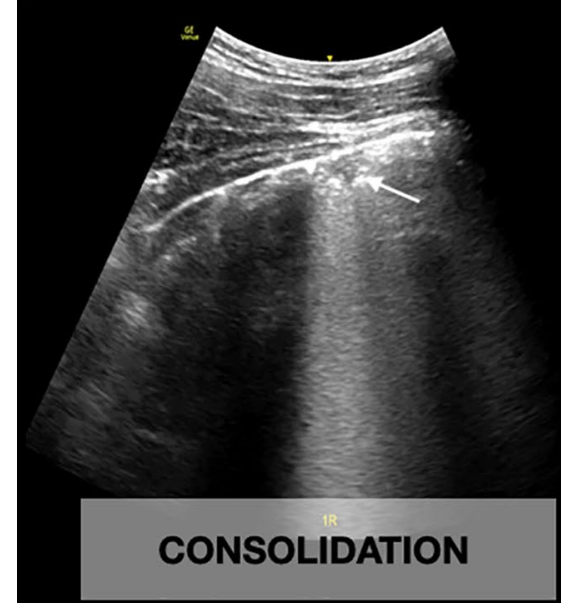

G

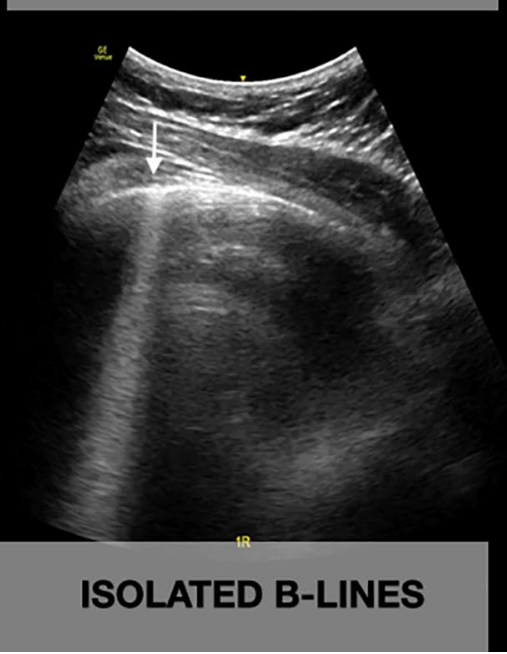

Fig. 3 Coronal (B) and sagittal (F) chest CT scan in lung window view of a 55 year-old woman, showing bilateral ground-glass opacifications correlated with lung ultrasound confluent B-lines (A, white

Table 5 Descriptive statistics and Interclass correlation coefficients (ICC) of each of the assessed Lung Ultrasonography (LUS) protocols

\begin{tabular}{llll}
\hline Protocol & Mean (SD) & ICC $(95 \%$ CI $)$ & $p$ value \\
\hline 8-zone protocol [3] & $40.8(23.3)$ & $0.353(-0.340-0.686)$ & 0.119 \\
10-zone protocol [5] & $55.3(20.1)$ & $0.470(0.018-0.733)$ & $\mathbf{0 . 0 2 2}$ \\
10-zone protocol [4] & $55.0(20.4)$ & $0.405(-0.121-0.697)$ & 0.052 \\
12-zone protocol [6] & $49.0(21.2)$ & $0.500(0.007-0.754)$ & $\mathbf{0 . 0 2 7}$ \\
12-zone protocol [7] & $59.8(20.0)$ & $0.427(0.095-0.711)$ & $\mathbf{0 . 0 2 2}$ \\
14-zone [8] & $53.9(20.5)$ & $0.493(0.018-0.745)$ & $\mathbf{0 . 0 1 9}$ \\
16-zone [9] & $55.5(21.1)$ & $0.425(0.08-0.707)$ & $\mathbf{0 . 0 4 1}$ \\
18-zone protocol [10] & $67.7(23.2)$ & $0.373(0.183-0.686)$ & $\mathbf{0 . 0 1 8}$ \\
72-zone protocol [11] & $58.9(19.9)$ & $0.476(0.042-0.742)$ & $\mathbf{0 . 0 1 1}$ \\
\hline
\end{tabular}

Statistically significant expressed in bold

STD standard deviation

reference LUS protocol (14-zone). The level of agreement of each protocol where $57 \%$ in the 8 -zone, $70 \%$ in the 10 -zone arrow), reticular pattern with isolated B-lines (G, white arrow), consolidations with air bronchogram $(\mathrm{D}$, white arrow) and normal lung with A-lines $(\mathrm{C}$, white arrow)

and $99 \%$ in the 12-zone. They concluded that a 12-zone scanning protocol had a similar performance comparable to the reference protocol (14-zone).

Similar to previously reported by Smargiassi [20], we found that limiting our exam to the anterior and lateral regions (8-zone) leads to an underestimation, whereas the posterior regions seem to be the most affected areas, where as proven, the examination of an isolated point per lung is not enough (10-zone protocol with one posterior point [4]). This study along with our results highlights the importance of scanning the posterior areas.

It is important to highlight that LUS reflects the lesions in the lung surface, and not necessary, the degree of the whole lung alteration. This might explain why incorporating more points in the exam might not translate into a higher accuracy of the lung involvement.

These findings are novel and useful, since they suggest that a 12-zone protocol could have a high degree of 
concordance with $\mathrm{CT}$, with similar performance to more comprehensive and time-consuming exams.

Moreover, we want to highlight that LUS is not an anatomical ultrasound, but a clinical-interpretation of artifacts, that should not replace chest X-ray or CT, instead complement them, and be regarded as its use as a clinical and diagnostic complementary and monitoring tool [21].

We acknowledge some study limitations. First of all, the poor specificity of LUS, thus the results from this study provides insights in COVID-19, and an opportunity to further investigate the role of LUS when the prevalence of disease decreases. Secondly, a small and convenience sample of confirmed COVID-19 patients, the expert sonographer performed all ultrasound scans on a consecutive sample selected based on his availability, which limits the generalizability of our results, and ought to be validated in future studies. Another limitation is that this study did not analyze the dynamic changes of the imaging modalities in different stages, and the patient outcomes, which would have a higher clinical impact. Therefore, for this purpose, we suggest the study can be considered hypothesis generating.

Although, these limitations are important, we believe that our study results support the current recommendation of a simple but comprehensive exam, including the superior and inferior areas of the anterior, lateral and posterior regions of each hemithorax. We consider appropriate a 12-zone exam protocols, as described in this study, and raise the question of the cost-effectiveness of more comprehensive exams.

\section{Conclusion}

In conclusion, our study results suggest that performing an ultrasound protocol with 12-zone scanning, including the superior and inferior areas of the anterior, lateral and posterior regions of the chest was consistent with higher ICC and higher degree of concordance with CT. We emphasize the need of a more standardization technique to further implement and develop this imaging modality in COVID-19.

Author contributions All authors have contributed to this work. Conception and design: YTC. Analysis and interpretation: YTC, MMG. Data collection: YTC, SOV, KAV, ADT, LPG, TVV, EMH, AGV, IAB, ATV, MMG. Writing the article: YTC, ATV. Critical revision of the article: YTC, SOV, KAV, ADT, LPG, TVV, EMH, AGV, IAB, ATV, MMG. Final approval of the article: YTC, SOV, KAV, ADT, LPG, TVV, EMH, AGV, IAB, ATV, MMG. Statistical analysis: YTC. Overall responsibility: YTC, MMG.

Funding This research received no external funding.
Data access and responsibility The principal investigator, Yale Tung Chen, had full access to all of the data in the study and takes responsibility for the integrity of the data and the accuracy of the data analysis.

\section{Declarations}

Conflict of interest The authors have declared no conflicts of interest.

Ethical approval All authors read and approved the final manuscript. This work has not been supported by public grants or financial support. No sources of funding were used to assist in the preparation of this study. Each author certifies that he has no commercial associations that might pose a conflict of interest in connection with the submitted article. We certify that this research was conducted in conformity with ethical principles of our institution. This work, figures and tables, have not been previously published and reproduced from another source.

\section{References}

1. Johns Hopkins Coronavirus Resource Center. https://coronavirus. jhu.edu/map.html. Accessed 9 Feb 2021.

2. Liu RB, Tayal VS, Panebianco NL, Tung-Chen Y, Nagdev A, Shah $S$ et al (2020) Ultrasound on the frontlines of COVID-19: report from an international webinar. Acad Emerg Med Off J Soc Acad Emerg Med 27(6):523-526

3. Moore S, Gardiner E. Point of care and intensive care lung ultrasound: a reference guide for practitioners during COVID-19. Radiography. 2020;S1078817420300572.

4. Clevert D-A. Lung ultrasound in patients with coronavirus COVID-19 disease. SIEMENS. https://www.siemens-healthinee rs.com/ultrasound/lung-ultrasound-covid-19. Accessed 29 Nov 2020

5. Volpicelli G, Lamorte A, Villén T (2020) What's new in lung ultrasound during the COVID-19 pandemic. Intensive Care Med 46(7): 1445-1448

6. Tung-Chen Y, Martí de Gracia M, Díez-Tascón A, AlonsoGonzález R, Agudo-Fernández S, Parra-Gordo ML, et al. Correlation between chest computed tomography and lung ultrasonography in patients with coronavirus disease 2019 (COVID-19). Ultrasound Med Biol. 2020;S030156292030301X.

7. Manivel V, Lesnewski A, Shamim S, Carbonatto G, Govindan T (2020) CLUE: COVID-19 lung ultrasound in emergency department. Emerg Med Australas 32(4):694-696

8. Soldati G, Smargiassi A, Inchingolo R, Buonsenso D, Perrone T, Briganti DF et al (2020) Proposal for international standardization of the use of lung ultrasound for patients with Covid-19: a simple, quantitative, reproducible method. J Ultrasound Med 39(7):1413-1419

9. Gargani L, Soliman-Aboumarie H, Volpicelli G, Corradi F, Pastore MC, Cameli M (2020) Why, when, and how to use lung ultrasound during the COVID-19 pandemic: enthusiasm and caution. Eur Heart J Cardiovasc Imaging 21(9):941-948

10. Vassalou EE, Karantanas AH, Antoniou KM (2020) Proposed lung ultrasound protocol during the COVID-19 outbreak. J Ultrasound Med 6:jum.15402

11. Gargani L, Doveri M, D’Errico L, Frassi F, Bazzichi ML, Sedie AD et al (2009) Ultrasound lung comets in systemic sclerosis: a chest sonography hallmark of pulmonary interstitial fibrosis. Rheumatology 48(11):1382-1387 
12. Hansell DM, Bankier AA, MacMahon H, McLoud TC, Müller NL, Remy J (2008) Fleischner society: glossary of terms for thoracic imaging. Radiology 246(3):697-722

13. Gargani L, Volpicelli G (2014) How I do it: lung ultrasound. Cardiovasc Ultrasound 12(1):25

14. Wu J, Wu X, Zeng W, Guo D, Fang Z, Chen L et al (2020) Chest CT findings in patients with coronavirus disease 2019 and its relationship with clinical features. Investig Radiol. 55(5):257-261

15. Koo TK, Li MY (2016) A guideline of selecting and reporting intraclass correlation coefficients for reliability research. J Chiropr Med 15(2):155-163

16. Allinovi M, Parise A, Giacalone M, Amerio A, Delsante M, Odone A et al (2020) Lung ultrasound may support diagnosis and monitoring of COVID-19 pneumonia. Ultrasound Med Biol 46(11):2908-2917

17. Vitale V, Rossi E, Di Serafino M, Minelli R, Acampora C, Iacobellis F, D'Errico C, Esposito A, Esposito F, Vallone G, Zeccolini M (2018) Pediatric encephalic ultrasonography: the essentials. J Ultrasound. https://doi.org/10.1007/s40477-018-0349-7
18. Minella R, Minelli R, Rossi E, Cremone G, Tozzi A (2020) Gastroesophageal and gastric ultrasound in children: the state of the art. J Ultrasound. https://doi.org/10.1007/s40477-020-00471-w

19. Mento F, Perrone T, Macioce VN, Tursi F, Buonsenso D, Torri E et al (2021) On the impact of different lung ultrasound imaging protocols in the evaluation of patients affected by coronavirus disease 2019: how many acquisitions are needed? J Ultrasound Med 40(10):2235-2238. https://doi.org/10.1002/jum.15580

20. Smargiassi A, Soldati G, Torri E, Mento F, Milardi D, Del Giacomo P et al (2021) Lung ultrasound for COVID-19 patchy pneumonia: extended or limited evaluations? J Ultrasound Med 40(3):521-528. https://doi.org/10.1002/jum.15428

21. Di Serafino M, Notaro M, Rea G et al (2020) The lung ultrasound: facts or artifacts? In the era of COVID-19 outbreak. Radiol med 125:738-753. https://doi.org/10.1007/s11547-020-01236-5

Publisher's Note Springer Nature remains neutral with regard to jurisdictional claims in published maps and institutional affiliations.

\section{Authors and Affiliations}

\section{Yale Tung-Chen ${ }^{1,6}$ - Silvia Ossaba-Vélez ${ }^{2} \cdot K^{-}$evin Stephen Acosta Velásquez ${ }^{2} \cdot$ Maria Luz Parra-Gordo $^{2}$. Aurea Díez-Tascón ${ }^{2} \cdot$ Tomás Villén-Villegas $^{3}$ · Esther Montero-Hernández ${ }^{1}$ - Andrea Gutiérrez-Villanueva ${ }^{1}$. Ángela Trueba-Vicente ${ }^{4} \cdot$ Isabel Arenas-Berenguer ${ }^{5} \cdot$ Milagros Martí de Gracia $^{2}$}

Silvia Ossaba-Vélez

silviaossaba@hotmail.com

Kevin Stephen Acosta Velásquez

kevinstephen.acosta@salud.madrid.org

Maria Luz Parra-Gordo

lparrag@telefonica.net

Aurea Díez-Tascón

aurea.diez@salud.madrid.org

Tomás Villén-Villegas

tomasvillen@gmail.com

Esther Montero-Hernández esthermhdez@hotmail.com

Andrea Gutiérrez-Villanueva

a.gutierrezv@gmail.com

Ángela Trueba-Vicente angelatrueba@gmail.com

Isabel Arenas-Berenguer isabelarbe@gmail.com
Milagros Martí de Gracia milagros.martidg@gmail.com

1 Department of Internal Medicine, Hospital Universitario Puerta de Hierro, Majadahonda, Madrid, Spain

2 Department of Emergency Radiology, Hospital Universitario de La Paz, Madrid, Spain

3 Facultad de Medicina, Universidad Francisco de Vitoria, Madrid, Spain

4 Department of Emergency Medicine, Hospital Universitario Ramón y Cajal, Madrid, Spain

5 Department of Emergency Medicine, Hospital Universitario La Paz, Madrid, Spain

6 Department of Medicine, Universidad Alfonso X El Sabio, Calle Joaquín Rodrigo, 1, Majadahonda, 28222 Madrid, Spain 